\title{
Ruído de Intermodulação na Banda Básica de Portadora FDM/FM que Compartilha um Repetidor Não Linear com Portadoras de Faixa Estreita
}

\author{
Raimundo Sampaio Neto e José Mauro P. Fortes
}

\begin{abstract}
Quando várias portadoras são transmitidas através de um canal não-linear, como - repetidor de um satélite, um grande número de produtos de intermodulação é gerado. Estes produtos constituem uma interferência que pode causar uma degradação substancial no desempenho do enlace. Em geral, a interferência produzida pela intermodulação contém produtos que envolvem a portadora interferida e que constituem, portanto, uma parcela interferente estatisticamente dependente do sinal desejado. Esta dependência \& muitas vezes desprezada em análises menos rigorosas dos efeitos de intermodulação. Este trabalho apresenta um desenvolvimento analítico que leva em consideração, de forma precisa, a dependência estatistica entre os sinais interferido e interferente e a filtragem da intermodulação pelo filtro de recepção da portadora desejada na determinação dos efeitos da intermodulação na banda básica de uma portadora FDM/FM que compartilha uma não-linearidade com várias portadoras de faixa estreita. Os resultados obtidos mostram que o tratamento adequado dos produtos de intermodulação dependentes pode levar a estimativas de razão sinal-ruldo de intermodulação atê $8 \mathrm{~dB}$ inferiores àquelas obtidas através de análises que consideram todos os produtos como independentes da portadora desejada.
\end{abstract}

\section{Introdução}

Quando várias portadoras são transmitidas através de um canal não-linear, como o repetidor de um satélite, um grande número de produ tos de intermodulação é gerado. Estes produtos constituem uma interferência que pode causar uma degradação substancial no desempenho do enlace. Uma análise exata dos efeitos de intermodulação pode ser bastante complexa. Entretanto, na maioria dos casos de interesse, os produtos de intermodulação ditos de terceira ordem constituem a parcela dominante da intermodulação total, permitindo que uma análise restrita a tais produtos forneça estimativas confiáveis de desempenho.

Em situações onde uma portadora analógica modulada em ângulo compartilha a não-linearidade com outras portadoras, é de interesse determinar os efeitos

Os autores são professores do Centro de Estudos em Telecomunicações da PUC/Rio, 22453, Rio de Janeiro RJ.

Revista da Socledade Brasllelra de Telecomunlcaçס̋es

Volume $4, \mathrm{~N} .^{\circ} 1$, dezembro 1989 
do ruído produzido pela intermodulação quando da demodulação do sinal desejado. Existem naturalmente produtos de terceira ordem que envolvem a portadora desejada e que não são totalmente filtrados pelo filtro de recepçào desta portadora. Embora em análises menos rigorosas esta dependência seja muitas vezes desprezada, ela deve ser considerada na determinação dos efeitos da intermodulação sobre a portadora desejada.

Uma análise que leva em consideração a dependência mencionada é apresentada em [1] para a situação em que portadoras com modulação em ângulo e amplitude constante compartilham a não-linearidade. Entretanto, a análise em [1] não considera a existência do filtro de recepção da portadora desejada, evitando assim a complexidade envolvida na análise do ruído de intermodulação gerado em banda básica por interferência dependente e parcialmente filtrada.

O presente trabalho apresenta um desenvolvimento analítico que leva em consideração, de forma precisa, a dependência estatística entre os sinais interferido e interferente e a filtragem da intermodulação pelo filtro de recepção da portadora desejada na determinação dos efeitos da intermodulação na banda básica de uma portadora FDM/FM que compartilha uma não-linearidade com várias portadoras de faixa estreita (SCPC). Foi também considerada a possível ativação por voz das portadoras SCPC. A existência de portadoras ativadas por voz provoca variações nas amplitudes complexas dos sinais na saída do dispositivo não-linear.

A análise se desenvolve na Seção 4 e é feita a partir de resultados apresentados nas seções 2 e 3 que tratam respectivamente da interferência sobre portadoras moduladas em ângulos e da caracterização dos sinais na saída do dispositivo não-linear. Na Seção 5 , os resultados analíticos obtidos na Seção 4 são aplicados a um exemplo numérico que considera uma configuração particular na qual uma portadora FDM/FM compartilha a não-linearidade com 500 portadoras SCPC/FM ativadas por voz. Neste exemplo, os efeitos da.intermodulação são determinados de duas maneiras distintas. Na primeira, todos os produtos de intermodulação são tratados como se fossem independentes e, na segunda, a parcela de produtos dependentes é isolada e tratada adequadamente. Como resultado, são apresentadas as razões sinal-ruído de intermodulação em cada canal do sinal multiplex da portadora desejada. Observa-se que o tratamento adequado dos produtos de intermodulação dependentes pode levar a estimativas de razão sinal-ruído de intermodulação até $8 \mathrm{~dB}$ inferiores àquelas obtidas através de análises menos rigorosas que consideram todos os produtos como independentes. Finalmente, na Seção 6 , são apresentadas conclusões relativas ao trabalho.

\section{Interferência Sobre Portadoras Moduladas em Ângulo}

Esta seçāo apresenta uma expressão aproximada para o ruído de fase na 
banda básica de uma portadora modulada em ângulo; provocada por um processo interferente passa-faixa, sob condições de razão portadora-interferência alta.

Sejam $s(t)$ e $u(t)$ respectivamente os sinais desejados e interferentes na entrada do demodulador de fase. Utilizando-se a representação complexa de sinais passa-faixa, estes sinais podem ser escritos como

$$
s(t)=\operatorname{Re}\left\{\tilde{s}(t) e^{j 2 \pi f_{0} t}\right\}=\operatorname{Re}\left\{A e^{j[\theta(t)+\lambda]} e^{j 2 \pi f_{0} t}\right\}
$$

e

$$
u(t)=\operatorname{Re}\left\{\tilde{u}(t) e^{j 2 \pi f_{0} t}\right\}
$$

onde $\tilde{s}(t)$ e ũ(t) são as envoltórias complexas dos dois sinais com relação à freqüência $f_{0}$ da portadora desejada. Em (1), $A$ e $\theta(t)$. representam, respectivamente, amplitude e modulação de fase do sinal desêjado e $\lambda$ é uma variável aleatória uniformemente distribuída em $(0,2 \pi]$.

O demodulador de fase fornece, como saída, a fase do sinal composto $s(t)$ $+u(t)$ ou, equivalentemente, a fase do sinal complexo

$$
\tilde{r}(t)=\tilde{s}(t)+\tilde{u}(t),
$$

ou ainda

$$
\tilde{r}(t)=\tilde{s}(t)[1+\tilde{z}(t)]
$$

onde

$$
\tilde{z}(t)=\frac{\tilde{u}(t)}{\tilde{s}(t)}=\frac{1}{A^{2}} \tilde{u}(t) \tilde{s}^{*}(t)
$$

Tem-se portanto na saída do demodulador

$$
\underline{\tilde{r}(t)}=\theta(\mathrm{t})+\lambda+\eta(\mathrm{t})
$$

onde $\tilde{\tilde{r}(t)}$ representa a fase de $\tilde{r}(t)$ e o ruido de fase $\eta(t)$ é dado por

$$
\eta(\mathrm{t})=\operatorname{arctg}\left[\frac{\operatorname{lm}[\tilde{z}(\mathrm{t})]}{1+\operatorname{Re}[\tilde{z}(t)]}\right]
$$

Sob a condiçao de razão portadora-interferência alta, tem-se com grande probabilidade

$$
\frac{|\tilde{u}(t)|}{A}=|\tilde{z}(t)|<<1 .
$$


Como $|\operatorname{Re}[\tilde{z}(t)]|$ e $|\operatorname{lm}[\tilde{z}(t)]|$ são ambos menores que $|\tilde{z}(t)|$, a condição (7) permite aproximar $\eta(t)$ por

$$
\eta(t) \cong \operatorname{arctg}\{\operatorname{Im}[\tilde{z}(t)]\}=\operatorname{Im}[\tilde{z}(t)]
$$

Considerando-se finalmente (8) e (4), obtém-se

$$
\eta(t)=\frac{1}{A^{2}} \operatorname{lm}\left[\tilde{\mathbf{s}}^{*}(\mathrm{t}) \tilde{\mathrm{u}}(\mathrm{t})\right]=
$$

A função autocorrelação do processo $\eta(t)$ é então dada por

$R_{\eta}(t, t+r)=E[\eta(t) \eta(t+\tau)]=$

$$
=\frac{1}{2 A^{4}} \operatorname{Re}\left\{E\left[\tilde{g}^{*}(t) \tilde{g}(t+\tau)\right]\right\}-\frac{1}{2 A^{4}} \operatorname{Re}\{E[\tilde{g}(t) \tilde{g}(t+\tau)]\}
$$

onde

$$
\tilde{g}(t)=\tilde{s}^{*}(t) \tilde{u}(t) .
$$

$\mathrm{Na}$ obtenção de (10) foi utilizada a identidade

$$
\operatorname{Im}(a) \cdot \operatorname{Im}(b)=\frac{1}{2} \operatorname{Re}\left(a^{*} b\right)-\frac{1}{2} \operatorname{Re}(a b)
$$

No caso em que os processos $\tilde{s}(t)$ e $\tilde{u}(t)$ são estatisticamente independentes, a expressão geral (10) se reduz a

$$
R_{\eta}(\tau)=\frac{1}{2 A^{4}} \operatorname{Re}\left\{R_{\tilde{g}}(\tau)\right\},
$$

correspondente a uma densidade espectral de potência dada por

$$
S_{\eta}(f)=\frac{1}{4 A^{4}}\left[S_{\tilde{g}}(f)+S_{\tilde{g}}(-f)\right]
$$

com

$$
S_{\tilde{g}}(f)=S_{\tilde{s}}(-f) * S_{\tilde{u}}(f)
$$

que é um resultado conhecido, encontrável, por exemplo, em [2].

\section{Caracterização dos Sinais na Saída da TWT}

Considera-se aqui a situação particular em que o sinal de entrada da não-linearidade consiste em uma portadora FDM/FM faixa larga de amplitude constante e M portadoras SCPC/FM e/ou SCPC/PSK de faixa estreita, podendo cada 
uma destas $M$ portadoras ser ou não ativada por voz. Neste caso, o sinal de entrada da não-linearidade pode ser caracterizado por

$$
e_{i}(t)=\operatorname{Re}\left\{\sum_{m=0}^{M} A_{m}(t) \exp \left\{j\left[2 \pi f_{0} t+\phi_{m}(t)+\lambda_{m}\right]\right\}\right\}
$$

onde $A_{0}(t)=A_{0}$ e $f_{0}$ correspondem à amplitude e à freqüência da portadora FDM/FM, respectivamente; e

$$
\phi_{m}(t)=\theta_{m}(t)+2 \pi\left(f_{m}-f_{0}\right) t
$$

sendo $\theta_{m}(t) o$ sinal que modula em fase a m-ésima portadora e $f_{m}$ a freqüência desta portadora.

O sinal de saída da não-linearidade correspondente ao sinal de entrada em (15) será expresso [3] por

$$
e_{0}(t)=\operatorname{Re}\left\{\begin{array}{c}
\left.\exp \left[j 2 \pi f_{0} t\right], \sum_{\substack{k_{0}, k_{1}, \ldots, k_{M}=-\infty \\
k_{0}+k_{1}+\ldots k_{M}=1}}^{\infty} M\left(k_{0}, k_{1}, \ldots k_{M^{\prime}}, t\right) \exp \left[j \sum_{m=0}^{M} k_{m}\left(\phi_{m}(t)+\lambda_{m}\right)\right]\right\} \\
\text { (17) }
\end{array}\right.
$$

com a amplitude complexa $M\left(k_{0}, k_{1}, \ldots k_{M}, t\right)$ dada por

$$
M\left(k_{0}, k_{1}, \ldots k_{M}, t\right)=\sum_{s=1}^{L} b_{s} \prod_{m=0}^{M} J_{k_{m}}\left(\alpha_{s} A_{m}(t)\right)
$$

onde $\left\{b_{s} ; s=1, \ldots, L\right\}$ são os coeficientes complexos da expansão da característica não-linear de transmissão da TWT em funçōes de Bessel [3] e $\alpha$ é um número real, escolhido a priori com vistas a tornar mais precisa a aproximação desta característica por um número finito de parcelas da expansão.

\section{Caracterização do Sinal Desejado}

No caso particular em questão, a portadora desejada (FDM/FM) é obtida de (17), fazendo-se $k_{0}=1$ e $k_{m}=0 ; m \neq 0$. Deste modo a envoltória complexa da portadora desejada na saída da não-linearidade é dada por

$$
\tilde{s}(t)=M_{D}(t) \cdot \exp \left[j\left(\theta_{0}(t)+\lambda_{0}\right)\right]
$$

onde

$$
M_{D}(t)=\sum_{s=1}^{L} b_{s} J_{1} \cdot\left(\alpha s A_{0}\right) \underset{m=1}{M} J_{0}\left(\alpha s A_{m}(t)\right)
$$


Quarido portadoras ativadas por voz estão também presentes no sistema, a amplitude complexa $\mathrm{M}_{\mathrm{D}}$, da envoltória complexa $\tilde{s}(\mathrm{t})$, possui variações temporais (conversão $A M / P M)$. Entretanto, a média estatística de $M_{D}(t)$, dada por

$\bar{M}_{D} \triangleq E\left[M_{D}(t)\right]=A_{D} e^{j \alpha_{D}}=\sum_{s=1}^{M} b_{s} J_{1}\left(\alpha_{s} A_{0}\right) \prod_{m=1}^{M}\left[p_{m} j_{0}\left(\alpha s A_{m}\right)+\left(1-p_{m}\right)\right]$

não depende de t. Em (21), a quantidade $P_{m} \triangleq P\left[A_{m}(t)=A_{m}\right]$ é igual a 1 para as portadoras de amplitude constante, e representa 0 fator de ativação por voz para as portadoras ativadas por voz.

É possivel decompor (19) na forma

$$
\tilde{s}(t)=A_{D} \exp \left[j\left(\theta_{0}(t)+\lambda_{0}+\alpha_{D}\right)\right]+\tilde{u}_{s}(t)
$$

onde

$$
\tilde{u}_{s}(t)=\left[M_{D}(t)-\bar{M}_{D}\right] \exp \left[j\left(\theta_{0}(t)+\lambda_{0}\right)\right]
$$

Em (22), a envoltória complexa $\tilde{s}(t)$ foi decomposta num sinal desejado sem distorção mais uma parcela de interferência $\tilde{u}_{s}(t)$ responsável por um ruído de fase que, de acordo com (9), é dado por

$$
\eta_{\tilde{u}_{s}}(t)=\frac{1}{A_{D}^{2}} \operatorname{Im}\left[\bar{M}_{D}{ }^{*}\left[M_{D}(t)-\bar{M}_{D}\right]\right]
$$

Para escrever (24), considerou-se que

$$
\frac{E\left[\left|\tilde{u}_{s}(t)\right|^{2}\right]}{A_{D}^{2}}=\frac{E\left[\left|M_{D}(t)-\bar{M}_{D}\right|^{2}\right]}{\left|\bar{M}_{D}\right|^{2}} \ll 1
$$

ou seja, que as variações de $M_{D}(t)$, devidas às portadoras ativadas por voz, em torno de seu valor médio $\bar{M}_{D}$ são pequenas em presença de $\bar{M}_{D}$. Esta condição é certamente satisfeita quando as amplitudes normalizadas das portadoras ativadas por voz são pequenas, o que ocorre quando o número total de portadoras (ativadas por voz ou não) compartilhando o transponder com a portadora FDM/FM é suficientemente grande $(>50)[4]$.

Observa-se que a faixa de freqüências ocupada pelo ruído em (24) é ditada por aquela correspondente a $M_{D}(t)$. Devido à baixa freqüênçia dos padrões "on-off" da ativação por voz, a densidade espectral de potência deste processo está concentrada em freqüência baixas, muito menores que a freqüência mínima do sinal multiplex (usualmente $12 \mathrm{kHz}$ ). Por este motivo, a parcela de ruído devido à conversão AM/PM pode ser desprezada e o sinal desejado em (22) pode ser aproximado por 


$$
\tilde{s}(t)=\bar{M}_{D} \exp \left[i\left(\theta_{0}(t)+\lambda_{0}\right)\right]=A_{D} \exp \left[j\left(\theta_{0}(t)+\lambda_{0}+\alpha_{D}\right)\right]
$$

\section{Caracterização dos Produtos de Intermodulação de $3 .^{a}$ Ordem}

Neste trabalho são considerados apenas os produtos de intermodulação de terceira ordem, do tipo $f_{a}+f_{b}-f_{c}$. A envoltória complexa $\tilde{v}_{a b c}(t)$, relativa à freqüencia de referência $f_{0}$, de um produto deste tipo pode ser ubtida fazendose, em (17) e (18), $k_{a}=1, k_{b}=1, k_{c}=-1$ e $k_{m}=0, m \neq a, b, c$ onde $a, b, c \in \in$ $\{0,1, \ldots, M\}$. Deste modo, obtém-se

$$
\tilde{v}_{a b c}(t)=B(a, b, c, t) \exp \left[j\left(\theta_{a}(t)+\theta_{b}(t)-\theta_{c}(t)+\lambda\right)\right]
$$

onde

$$
B(a, b, c, t)=M(a, b, c, t) \exp \left[j 2 \pi\left(f_{a}+f_{b}-f_{c}-f_{0}\right) t\right]
$$

e

$$
\lambda=\lambda_{\mathrm{a}}+\lambda_{\mathrm{b}}-\lambda_{\mathrm{c}}
$$

com

$$
\begin{aligned}
& M(a, b, c, t)=\sum_{s=1}^{L} b_{s} J_{1}\left(\alpha s A_{a}(t)\right) J_{1}\left(\alpha_{s} A_{b}(t)\right) J_{-1} ;\left(\alpha_{s} A_{c}(t)\right) . \\
& \prod_{\substack{m=0 \\
m \neq a, b, c}}^{M} J_{0}\left(\alpha s A_{m}(t)\right)
\end{aligned}
$$

Observe que os processos $M(a, b, c, t)$ e $\theta_{a}(t)+\theta_{b}(t)-\theta_{c}(t)$ não são necessariamente independentes. A dependência entre eles existirá, se pelo menos uma das portadoras envolvidas no produto for ativada por voz. Entretanto, pode ser verificado que, em qualquer caso, a função autocorrelaçāo do processo $\tilde{v}_{a b c}(t)$ pode ser expressa na. forma

$$
\mathrm{R}_{\tilde{v}_{\mathrm{abc}}}(\tau)=\rho_{\mathrm{a}}(\tau) \rho_{\mathrm{b}}(\tau) \rho_{\mathrm{c}}^{*}(\tau) \mathrm{D}_{\mathrm{abc}}(\tau) \exp \left[\mathrm{j} 2 \pi\left(\mathrm{f}_{\mathrm{a}}+\mathrm{f}_{\mathrm{b}}-\mathrm{f}_{\mathrm{c}}-\mathrm{f}_{0}\right) \tau\right]
$$
com

$$
\begin{array}{r}
D_{a b c}(\tau)=p_{a} p_{b} p_{c} \sum_{s=1}^{L} \sum_{n=1}^{L} b_{s}^{*} b_{n} J_{1}\left(\alpha s A_{a}\right) J_{1}\left(\alpha n A_{a}\right) J_{1}\left(\alpha s A_{b}\right) \\
J_{1}\left(\alpha n A_{b}\right) J_{1}\left(\alpha s A_{c}\right) J_{1}\left(\alpha n A_{c}\right) .
\end{array}
$$

$$
\sum_{\substack{m=0 \\ m \neq a, b, c}}^{M} E\left[J_{0}\left(\alpha s A_{m}(t)\right) J_{0}\left(\alpha n A_{m}(t+\tau)\right)\right] .
$$


e

$$
p_{k}=P\left[A_{k}(t)=A_{k}\right] \quad, \quad k=a, b, c
$$

e onde $\rho_{k}(\tau), k=a, b, c$ corresponde à função autocorrelação normalizada $\left\langle\rho_{k}(0)=1\right)$ da envoltória complexa de cada uma das portadoras envolvidas no produto, na entrada da não-linearidade. Levou-se em consideração ainda que $J_{-1}(x)=-J_{1}(x)$.

\section{Ruído Devido à Intermodulação na Banda Básica de uma Portadora $\mathrm{FDM} / \mathrm{FM}$}

Analisa-se inicialmente o efeito, na banda básica de uma portadora FDM/FM com envoltória complexa s̃(t) dada por (26), provocado por um produto de intermodulação de terceira ordem cuja envoltória complexa com relação a $f_{0}$ é da forma dada em (27). Em seguida, o caso de uma soma de produtos de intermodulação da forma acima é analisado e a correlação entre as parcelas de ruído por eles geradas é eonsiderada,

Considera-se ainda nesta análise, o efeito do filtro de recepção $H(f)$ da portadora FDM/FM. Neste caso, o sinal interferente na entrada do demodulador de fase é um sinal passa-fixa com envoltória complexa dada por

$$
\tilde{u}(t)=\tilde{h}(t) * \tilde{v}_{a-b c}(t)
$$

onde $\tilde{h}(t)$ é a resposta ao impulso do equivalente passa-baixo do filtro de recepção $H(f)$ e $\tilde{v}_{a b c}(t)$ é dado por (27).

Note-se que o resultado em (14) pode ser aplicado sempre que os sinais interferido e interferente forem estatisticamente independentes. Nestes casos, a consideração do filtro de recepção não introduz dificuldades significativas no tratamento analítico do problema. Entretanto, este não é necessariamente o caso quando a interferência é produzida por intermodulação. Na verdade, a portadora interferida pode também participar na composição de produtos de intermodulação que não são totalmente filtrados pelo filtro de recepção, criando desta maneira uma parcela interferente estatisticamente dependente da portadora interferida. Em muitos casos, esta dependência não pode ser desprezada a priori. A análise desta situação pode ser feita a partir da expressão geral (10).

\subsection{Produtos de Intermodulação Dependentes e Filtrados}

Considera-se que o sinal interferente, cuja envoltória complexa é dada por (33) e (27), é dependente da portadora FDM/FM desejada. Isto significa 
que esta portadora participa da formação do produto de intermodulação em questão. Deste modo tem-se um dos índices, a, b ou c em (27) igual a zero. São identificados então dois casos, dependendo do sinal com o qual a fase da portadora interferida entra na composição da fase do produto de intermodulação. No primeiro caso (caso I) considera-se $\left(a=0, b=i_{1}, c=k\right)$ ou $(b=0, a=i, c=k)$ e no segundo (caso II) considera-se que $(a=i, b=k, c=0)$. As envoltórias complexas dos sinais interferentes associados a cada um destes casos, obtidas de (33), (27) e (28) são dadas por

$$
\tilde{u}_{ \pm}(t)=e^{j \lambda_{ \pm}}\left\{\tilde{h}(t) * B_{ \pm}(t) \exp \left[j\left( \pm \theta_{0}(t)+\theta_{i}(t) \pm \theta_{k}(t)\right)\right]\right\}
$$

onde

$$
\lambda_{ \pm}= \pm \lambda_{0}+\lambda_{i} \pm \lambda_{k}
$$

e

$$
B_{ \pm}(t)=M(0, i, k, t) \exp \left[j 2 \pi\left( \pm f_{0}+f_{j} \pm f_{k}-f_{0}\right) t\right] .
$$

Em (34) e (35), a expressão relativa ao caso I é obtida tomando-se os sinais superiores e a relativa ao caso II tomando-se os sinais inferiores.

\section{Existência de produtos correspondentes ao caso I e ao caso II}

Observa-se de (31) que a banda do espectro do sinal interferente a ser filtrado é da ordem da banda da portadora FDM/FM (e portanto da banda de $\tilde{h}(t))$, já que as duas outras portadoras $i$ e $k$ que compõem o produto de intermodulação são de faixa estreita. Considerando-se ainda que este espectro está centrado na freqüência $f_{i}-f_{k}$ no caso I e na freqüência $f_{i}+f_{k}-2 f_{0}$ no caso II, conclui-se que quando as portadoras de faixa estreita estiverem todas localizadas em um mesmo lado (à esquerda ou à direita) da portadora FDM/FM, todos os produtos incluídos no caso II serão rejeitados pelo filtro de recepção. Caso contrário, tanto produtos do caso I como do caso II poderão cair na faixa do filtro de resposta $\tilde{h}(t)$.

$\mathrm{Na}$ determinação de $R_{\eta}(\tau)$, devido à presença de fases aleatórias no sinal $\tilde{g}(t)$, a segunda parcela em (10) é nula. Deste modo, (13) e (14a) podem ser utilizadas. Entretanto, devido à dependência entre os sinais interferentes e interferido, (14b) não é válida.

Determinação de $R_{\tilde{g}}(\tau)$

A partir de (11) e (34), tem-se

$$
R_{\tilde{\tilde{g}}}^{ \pm}(\tau)=E\left[\tilde{s}^{*}(t) \tilde{u}_{ \pm}(t) \tilde{s}(t+\tau) \tilde{u}_{ \pm}{ }^{*}(t+\tau)\right]
$$




$$
\begin{aligned}
& =E\left\{\int _ { - \infty } ^ { \infty } \int _ { - \infty } ^ { \infty } \tilde { s } ^ { * } ( t ) \tilde { s } ( t + \tau ) \tilde { h } ( \alpha ) B _ { \pm } ( t - \alpha ) \operatorname { e x p } \left[j\left( \pm \theta_{0}(t-\alpha)+\theta_{i}(t-\alpha) \mp \theta_{k}(t-\alpha)\right]\right.\right. \\
& \left.. h^{*}(\beta) B_{ \pm}^{*}(t+\tau-\beta) \exp \left[j\left(\mp \theta_{0}(t+\tau-\beta)-\theta_{i}(t+\tau-\beta) \pm \theta_{k}(t+\tau-\beta)\right)\right] d \alpha d \beta\right\}
\end{aligned}
$$

ou ainda levando-se (26) em consideraçăo

$$
\begin{aligned}
R_{\tilde{g}}^{ \pm}(\tau)=A_{D}^{2} \int_{-\infty}^{\infty} \int_{-\infty}^{\infty} \tilde{h}(\alpha) \tilde{h}^{*}(\beta) R_{B i k}^{ \pm}(\beta-\alpha-\tau) \\
E\left\{\exp \left[j\left(\theta_{0}(t+\tau)-\theta_{0}(t) \pm \theta_{0}(t-\alpha) \mp \theta_{0}(t+\tau-\beta)\right)\right]\right\} d \alpha d \beta
\end{aligned}
$$

onde

$R_{B i k}^{ \pm}(\tau)=E\left\{B_{ \pm}^{*}(t) B_{ \pm}(t+\tau) \exp \left[j\left(\theta_{i}(t+\tau)-\theta_{1}(t)\right)\right] \exp \left[j\left(\mp \theta_{k}(t+\tau) \pm \theta_{k}(t)\right)\right]\right\}$

Para escrever (37), levou-se em consideração que os processos $\theta_{0}(t)$ e $B_{ \pm}(t)$ são estatisticamente independentes. Tal condição nao foi supostà no caso dos pares $\theta_{i}(t), B_{ \pm}(t)$ e $\theta_{k}(t), B_{ \pm}(t)$, devido à possibilidade de $\theta_{i}(t)$ e $\theta_{k}(t)$ corresponderam a portadoras ativadas por voz.

A determinação do valor esperado em (37) pode ser obtida utilizando-se a aproximação usual de que o sinal multiplex que modula a portadora FDM/FM pode ser modelado por um processo Gaussiano, o que significa que o processo $\theta_{0}(t)$ é também Gaussiano. Sob estas condições, resulta para $R_{\tilde{g}}^{ \pm}(t)$ a expressão [5]

$$
\begin{array}{r}
R_{\tilde{g}}^{ \pm}(\tau)=A_{D}^{2} \int_{-\infty}^{\infty} \int_{-\infty}^{\infty} \tilde{h}(\alpha) \tilde{h}^{*}(\beta) R_{B i k}^{ \pm}(\beta-\alpha-\tau) \rho_{0}(\beta-\alpha-\tau) \rho_{0}(\tau) \\
{\left[\frac{\rho_{0}(\alpha)}{\rho_{0}(\tau+\alpha)} \cdot \frac{\rho_{0}(\beta)}{\rho_{0}(\tau-\beta)}\right] \mathrm{d} \alpha \mathrm{d} \beta}
\end{array}
$$

onde $\rho_{0}(\tau)$ representa a função autocorrelação normalizada da envoltória complexa da portadora FDM/FM, na entrada da não linearidade.

Para a determinação de $R_{B_{i k}}^{+}($(.), note-se de $(38)$, que esta função representa a autocorrelação do processo de entrada do filtro de recepção em (34) 
(caso I), fazendo-se $\theta_{0}(\mathrm{t}) \equiv 0$ Esta autocorrelação pode portanto ser obtida de (31) e (32), fazendo-se $\rho_{0}(\tau)=\mathrm{p}_{\mathrm{a}}=1, \mathrm{a}=0, \mathrm{~b}=\mathrm{i}, \mathrm{c}=\mathrm{k}$.Procedendo-se de forma análoga para $\mathrm{R}_{\mathrm{Bik}}^{-}($.$) , obtém-se$

$$
R_{\text {Bik }}^{ \pm}(\tau)=\rho_{i}(\tau) \rho_{k}(\tau) D_{0 i k}(\tau) \exp \left[j 2 \pi\left( \pm f_{0}+f_{i} \mp f_{k}-f_{0}\right) \tau\right]
$$

onde por simplicidade foi considerado que os espectros das portadoras são simétricos em relação às suas freqüências centrais, e portan to as autocorrelações $\rho_{i}(\tau), i=0, \ldots, M$, são reais.

Observa-se que o resultado em (39) é válido para portadoras FDM/FM cujo sinal modulador possa ser aproximado por um processo Gaussiano, independentemente da forma do seu espectro. Será agora considerada a aproximação Gaussiana para a forma do espectro de uma portadora FDM/FM de faixa larga. Esta aproximação permitirá a determinação do espectro da intermodulação em banda básica de uma forma bem mais tratável do que a partir de (39).

\section{Caso Particular: Portadora FDM/FM com Espectro Gaussiano}

Neste caso, o espectro normalizado da envoltória complexa da portadora FDM/FM é dado por

$$
\bar{S}_{0}(f)=\frac{1}{\sqrt{2 \pi} d_{f}} \exp \left(-\frac{f^{2}}{2 d_{f}^{2}}\right)
$$

onde

$$
d_{f}=\left[\int_{-\infty}^{\infty} f^{2} \bar{S}_{0}(f) d f\right]^{1 / 2}
$$

é o desvio r.m.s. ("root mean square") de freqüência da portadora, em Hz. Assim,

$$
\rho_{0}(\tau)=\mathcal{F}^{-1}\left[\bar{S}_{0}(f)\right]=\exp \left(-\frac{\tau^{2} d_{f}^{2}}{2}\right)
$$

Com $\rho_{0}(\tau)$ dado por (43), (39) pode ser simplificada, obtendo-se para o caso 1 ,

$$
R_{\tilde{g}}^{+}(\lambda)=A_{D}^{2} \int_{-\infty}^{\infty} R_{B i k}^{+}(\lambda \cdots \tau) \rho_{0}(\lambda) q_{u}(\lambda) d \lambda
$$

onde

$$
q(\lambda)=\tilde{h} *(\lambda) * \tilde{h}(-\lambda)
$$


- ou, tomando-se a transformada de Fourier de (44),

$$
S_{\tilde{g}}^{+}(-f)=A_{D}^{2} S_{B i k}^{+}(f) \quad\left[\bar{S}_{0}(f) *|\tilde{H}(f)|^{2}\right]
$$

com

$$
\mathrm{S}_{\text {Bik }}^{+}(f)=\mathcal{F}\left[R_{\text {Bik }}^{+}(\tau)\right]
$$

e $\tilde{H}(f)$ é a resposta de freqüencia do equivalente passa-baixa do filtro de recepção da portadora FDM/FM.

Para o caso II, obtém-se[5]

$$
R_{\tilde{g}}^{-}(\tau)=A_{D}^{2} \int_{-\infty}^{\infty} R_{B i k}^{-}(\lambda-\tau) \rho_{0}(\lambda--2 \tau) q(\lambda) d \lambda
$$

cuja transformada de Fourier fornece

$$
S_{\tilde{g}}^{-}(f)=A_{D}^{2} \int_{-\infty}^{\infty} S_{B i k}^{-}\langle\alpha) S_{0}^{\prime}(f+\alpha) Q^{\prime}(f-\alpha) d \alpha
$$

com

$$
Q^{\prime}(f)=\left|\tilde{H}\left(\frac{f}{2}\right)\right|^{2}
$$

e

$$
S_{0}^{\prime}(f)=\frac{1}{2} \bar{S}_{0}\left(-\frac{f}{2}\right)
$$

4.2. Efeito da Soma de Produtos de Intermodulação

Sob a condição de razão portadora-interferência alta, o ruído total em banda básica provocado pela soma de produtos de intermodulação é obtido, a partir de (9), através da soma das contribuições individuais, ou seja,

$$
\eta(t)=\frac{1}{A_{D}^{2}} \sum_{n} \eta_{n}(t)
$$

onde

$$
\eta_{n}(t)=\operatorname{Im}\left[\tilde{g}_{n}(t)\right]
$$




$$
\tilde{g}_{n}(t)=\tilde{s}^{*}(t) \tilde{u}_{n}(t)
$$

sendo $\tilde{u}_{n}(t)$ um produto filtrado da forma expressa por (34).

Embora todos os produtos de intermodulação sejam processos ortogonais (devido às fases aleatórias distintas), seus correspondentes em banda básica $\left\{\eta_{n}(t)\right\}$ não o são necessariamente. Isto significa que a obtenção do espectro de $\eta(t)$ não pode ser feita simplesmente através da soma dos espectros de $\eta_{n}(t)$, e as densidades espectrais cruzadas devem ser consideradas.

Uma análise cuidadosa de (34) e (52), levando em consideração a presença das fases aleatórias $\lambda_{ \pm}$, permite concluir que:

i) as parcelas de (52a) geradas por produtos de intermodulação correspondentes ao caso II são todas ortogonais entre si, e ortogonais àquelas geradas por produtos de intermodulação correspondentes ao caso l;

ii) cada uma das parcelas de (52a) geradas por produtos de intermodulação correspondentes ao caso l é correlatada com uma, e apenas uma, das demais parcelas geradas por tais produtos (esta correlação se dá entre parcelas geradas por produtos com freqüências centrais $\left.f_{0}+f_{i}-f_{k} \in f_{0}+f_{k}-f_{j}\right)$.

Considerando-se estas observações, o espectro total do ruído em banda básica gerado por produtos correspondentes ao caso I pode ser obtido através da soma dos espectros das parcelas de ruído associadas a pares de produtos da forma identificada na observação (ii) acima. Seja então ũ(t) $=\tilde{u}_{1}(t)+\tilde{u}_{2}(t)$, onde $\tilde{u}_{1}(t)$ e $\tilde{u}_{2}(t)$ caracterizam um par de produtos de intermodulação filtrados, satisfazendo a relação mencionada em (ii). Para um dado par (i, $k$ ), tem-se a partir de (34) e (35) que

$\tilde{u}_{1}^{+}(t)=e^{j\left[\lambda_{0}+\lambda_{i}-\lambda_{k}\right]} \quad \tilde{h}(t) * M(0, i, k, t) e^{j\left[2 \pi\left(f_{i}-f_{k}\right) t+\left[\theta_{0}(t)+\theta_{i}(t)-\theta_{k}(t)\right)\right]}$

enquanto $\tilde{u}_{2}^{+}(t)$ é obtido permutando-se os índices i e k em (53).

A parcela de ruído associada a ũ(t) é dada por

$$
\eta_{i, k}(t)=\eta_{1}(t)+\eta_{2}(t)
$$

onde

e

$$
\eta_{\ell}(t)=\frac{1}{A_{D}^{2}} \operatorname{Im}\left[\tilde{g}_{\ell}(t)\right]
$$

$$
\tilde{g}_{\ell}(t)=\tilde{s}^{*}(t) \tilde{u}_{\ell}^{+}(t) \quad, \quad \ell=1,2
$$


A densidade espectral de potência de $\eta_{i, k}(t)$ é então dada por

$$
S_{\eta_{i, k}}(f)=S_{\eta_{1}}(f)+S_{\eta_{2}}(f)+S_{\eta_{1} \eta_{2}}(f)+S_{\eta_{1} \eta_{2}}(-f)
$$

As duas primeiras parcelas de (57) podem ser obtidas utilizando-se (14a) e a transformada de Fourier de (39), que no caso de espectro Gaussiano para a portadora FDM/FM é dada por (46). Por outro lado, a determinação de $S_{\eta_{1} \eta_{2}}(f)$ é obtida a partir da correlação $R_{\eta_{1} \eta_{2}}(\tau)$, dada por

$R_{\eta_{1} \eta_{2}}(\tau)=\frac{1}{2 A_{D}^{4}} \operatorname{Re}\left\{E\left[\tilde{g}_{1}^{*}(t) \tilde{g}_{2}(t+\tau)\right]\right\}-\frac{1}{2 A_{D}^{4}} \operatorname{Re}\left\{E\left[\tilde{g}_{1}(t) \tilde{g}_{2}(t+\tau)\right]\right\}$

Considerando-se as fases aleatórias presentes em $\tilde{u}^{+}(t)$ e $\tilde{u}^{+}(t) \cdot(56)$ e a expressão para $\tilde{s}(\mathrm{t})$ dada por $(26)$, é fácil verificar que a primeira parcela do segundo membro de (58) é nula. Deste modo tem-se

$$
\mathrm{R}_{\eta_{1} \eta_{2}}(\tau)=-\frac{1}{2 A_{D}^{4}} \operatorname{Re} \cdot\left[\mathrm{R}_{\tilde{g}_{1} \tilde{g}_{2}}(\tau)\right]
$$

onde

$$
R_{\tilde{g}_{1} \tilde{g}_{2}}(\tau) \triangleq E\left[\tilde{g}_{1}(t) \tilde{g}_{2}(t+\tau)\right]
$$

De (59) resulta

$$
S_{\eta_{1} \eta_{2}}(f)=-\frac{1}{4 A_{D}^{4}}\left[S_{\tilde{g}_{1} \tilde{g}_{2}}(f)+S_{\tilde{g}_{1} \tilde{g}_{2}}^{*}(-f)\right]
$$

onde

$$
\mathrm{S}_{\tilde{g}_{1} \tilde{g}_{2}}(f)=\mathcal{F} \quad\left[R_{\tilde{g}_{1} \tilde{g}_{2}}(t)\right]
$$

A partir de (57), (14a) e (61) obtém-se finalmente

$$
S_{\eta_{i, k}}(f)=F(f)+F(-f)
$$

onde

$$
F(f)=\frac{1}{4 A_{D}^{4}} \quad\left\{\left[S_{\tilde{g}_{1}}(f)-S_{\tilde{g}_{1} \tilde{g}_{2}}(f)\right]+\left[S_{\tilde{g}_{2}}(-f)-S_{\tilde{g}_{1} \tilde{g}_{2}}^{*}(f)\right]\right\}
$$

Jeterminação de $\mathbf{R}_{\tilde{\mathbf{g}}_{1} \tilde{g}_{2}}(\gamma)$

Zonsiderando-se $(60),(56)$ e as definiçōes de $\tilde{s}(t), \tilde{u}_{1}^{+}(t)$ e $\tilde{u}_{2}^{+}(t)$ dadas em 26) e (53), é possivel escrever

36 


$$
\begin{aligned}
R_{\tilde{g}_{1} \tilde{g}_{2}}(\tau)=A_{D}^{2} e^{-j 2 \alpha_{D}} \int_{-\infty}^{\infty} \int_{-\infty}^{\infty} \tilde{h}(\alpha) \tilde{h}(\beta) R_{B i k}^{\prime}(\beta-\alpha-\tau) E\left\{\operatorname { e x p } \left[j \left(\theta_{0}(t-\alpha)-\theta_{0}(t)+\right.\right.\right. \\
\left.\left.\left.+\theta_{0}(t+\tau-\beta)-\theta_{0}(t+\tau)\right)\right]\right\} \operatorname{d} \alpha d \beta
\end{aligned}
$$

onde

$R_{B i k}^{\prime}(\tau)=E\left\{M(0, i, k, t) M(0, i, k, t+\tau) e^{j 2 \pi\left(f_{i}-f_{k}\right) \tau} \theta^{j\left[\theta_{i k}(t+\tau)-\theta_{i k}(t)\right]}\right\}$

com

$$
\theta_{i k}(t)=\theta_{i}(t)-\theta_{k}(t)
$$

Observa-se que (65) e (66) são semelhantes a (37) e (38).

A determinação do valor esperado em (65) pode ser feita de forma inteiramente análoga àquela que permitiu a obtenção de (39) a partir de (37). Procedendo-se desta maneira, chega-se a[5]

$$
\begin{aligned}
R_{\tilde{g}_{1} \tilde{g}_{2}}(\tau)=A_{D}^{2} e^{-j 2 \alpha} \int_{-\infty-\infty}^{\infty} \int_{-\infty}^{\infty} \tilde{h}(\alpha) \tilde{h}(\beta) R_{B i k}^{\prime}(\beta-\alpha-\tau) \\
\frac{\rho_{0}(\alpha) \rho_{0}(\beta) \rho_{0}(\beta-\tau) \rho_{0}(\alpha+\tau)}{\rho_{0}(\beta-\alpha-\tau) \rho_{0}(\tau)} \mathrm{d} \alpha \mathrm{d} \beta
\end{aligned}
$$

que é similar a (39). A partir de (66) obtém-se, como em (40)

$$
R_{B i k}^{\prime}(\tau)=\rho_{i}(\tau) \rho_{k}(\tau) D_{O i k}^{\prime}(\tau) \exp \left[j 2 \pi\left(f_{i}-f_{k}\right) \tau\right]
$$

onde $D_{\text {Oik }}^{\prime}(\tau)$ é obtido substituindo-se, em (32), $b_{s}^{*}$ por $b_{s}$.

\section{Caso Particular: Portadora FDM/FM com Espectro Gaussiano.}

Neste caso, $\rho_{0}(\tau)$ é dado por (43), e (67) pode ser escrita na forma [5]

$$
R_{\tilde{g}_{1} \tilde{g}_{2}}(\tau)=A_{D}^{2} e^{-j 2 \alpha_{D}}\left[w(\tau) * R_{B i k}^{\prime}(-\tau)\right]
$$

onde 


$$
w(\tau)=\int_{-\infty}^{\infty} \tilde{h}\left(\lambda-\frac{\tau}{2}\right) \tilde{h}\left(\lambda+\frac{\tau}{2}\right) \rho_{0}(2 \lambda) d \lambda .
$$

Tomando-se a Transformada de Fourier de (69), obtém-se então

$$
S_{\tilde{g}_{1} \tilde{g}_{2}}(f)=A_{D}^{2} e^{-j 2 \alpha_{D}} \cdot W(f) S_{B i k}^{\prime}(-f)
$$

onde $S_{B j k}^{\prime}(f)$ é a transformada de Fourier de $R_{B i k}^{\prime}(\tau)$ e $W(f)$, obtida a partir de (70), é dada por

$$
w(f)=\mathcal{F}[w(\tau)]=\int_{-\infty}^{\infty} \tilde{H}(f+\beta) \bar{S}_{0}(\beta) \tilde{H}(f-\beta) d \beta
$$

$\operatorname{com} \vec{S}_{0}(f)$ dado por (41).

4.3. A Influência da Ativação por Voz na Forma de $S_{B i k}^{ \pm}(f) e S_{B i k}^{\prime}(f)$

Para uma portadora FDM/FM com espectro Gaussiano, (63), (64), (46) e (71) permitem calcular a densidade espectral de potência do ruído de fase Jevido a pares formados por produtos de intermodulação correspondentes ao caso I e localizados simetricamente com relaçāo à freqüência da portadora FDM/FM. No caso de produtos correspondentes ao caso II o espectro de interesse é obtido a partir de (14) e (49). Esta subseção considera a determinação das quantidades $S_{B i k}{ }^{ \pm}(f)$ e $S^{\prime}{ }_{B i k}(f)$, utilizadas em (46), (49) e (71).

A partir de (40) obtém-se

$$
S_{B i k}^{ \pm}(f)=\left[\bar{S}_{i} * \bar{S}_{k} * S_{D i k}\right]\left(f-\Delta f_{i k}^{ \pm}\right)
$$

Inde $\mathrm{S}_{\mathrm{Dik}}(f)=\mathcal{F}\left[\mathrm{D}_{\mathrm{Oik}}(\tau)\right]$ com $\mathrm{D}_{\mathrm{Oik}}(\tau)$ obtido de $(32)$, e

$$
\Delta f_{i k}^{ \pm}= \pm f_{0}+f_{i} \mp f_{k}-f_{0}
$$

tnalogamente, a partir de (68) tem-se

$$
S_{B i k}^{\prime}(f)=\left[\bar{S}_{i} * \bar{S}_{k} * S_{D i k}^{\prime}\right]\left(f-\Delta f_{i k}^{+}\right)
$$

Inde $\quad S_{D_{i k}}^{\prime}=\mathcal{F}\left[D_{0 i k}^{\prime}(\tau)\right]$ e $\Delta f_{i k}^{+}$é dado por (74). 
Com relação ao fator de distorção $D_{0 i k}(\tau)$, as seguintes observações podem ser feitas:

i) $D_{0 i k}(\tau)$ tem simetria conjugada, ou seja, $D_{0 i k}^{*}(\tau)=D_{0 i k}(-\tau) \quad$. Conseqüentemente $\mathrm{S}_{D i k}(\mathfrak{f})$ é função real;

ii) considerando-se a decomposição

$$
D_{0 i k}(\tau)=P_{0 i k}\left[\frac{D_{0 i k}(\infty)}{P_{0 i k}}+\frac{D_{0 i k}(\tau)-D_{0 i k}(\infty)}{P_{0 i k}}\right]
$$

onde

$$
P_{0 i k}=D_{0 i k}(0)=E\left[|M(0, i, k, t)|^{2}\right],
$$

observa-se que o espectro normalizado $S_{D i k}(\mathfrak{f}) / P_{0 i k}$ possui uma componente impulsional de área $\lambda=D_{0 i k}(\infty) / P_{0 i k}$ e uma componente não impulsional, cuja existência é devida à presença de portadoras SCPC/FM ativadas por voz, de área $1-\lambda$. Esta última parcela possui uma banda limitada por um valor da ordem de $m \cdot b_{0}$, onde $m$ é o número de portadoras ativadas por voz presentes no sistema, e b $b_{0}$ é a banda do processo "on-off" de ativação por voz que é da ordem de $2 \mathrm{~Hz}$ [4].

iii) Do exposto em (ii), tem-se que para valores pequenos de $m, S_{D i k}$ (f) irá existir somente em uma faixa de freqüências muito pequena quando comparada à faixa de $\overline{\mathrm{S}}_{i}(\mathrm{f}) * \overline{\mathrm{S}}_{\mathrm{k}}(\mathrm{f})$. Por outro lado, para valores razoáveis de $m(m>50)$, apesar da componente não impulsional de $S_{D i k}$ (f) existir numa banda de frequêencias que pode ser considerada significativa, sua potência é desprezível em presença da componente impulsional, já que $\lambda=1$ para valores grande de $m$ [4]. Conclui-se então que para efeitos da convolução em (73), $\mathrm{S}_{D_{\text {Dik }}}$ (f) pode ser considerado como um impulso de área $\mathrm{P}_{\mathrm{Oik}^{\prime}}$ ou seja,

$$
S_{B i k}^{ \pm}(f) \cong P_{0 i k}\left[\bar{S}_{i} * \bar{S}_{k}\right]\left(f-\Delta f_{i k}^{ \pm}\right)
$$

Com relação ao fator de distorção $D_{0 i k}^{\prime}(\tau)$, observações similares às dos itens (ii) e (iii) conduzem à aproximação

$$
S_{B i k}^{\prime}(f) \cong P_{0 i k}^{\prime}\left[\bar{S}_{i} * \bar{S}_{k}\right]\left(f-\Delta f_{i k}^{+}\right)
$$

onde $\mathrm{P}_{\mathrm{O} i \mathrm{k}}^{\prime}$ é a quantidade complexa dada por 


$$
P_{0 i k}^{\prime}=D_{0 i k}^{\prime}(0)=E\left\{[M(0, i, k, t)]^{2}\right\}
$$

4.4. A Influência do Filtro de Recepção no Espectro do Ruído de Fase

As expressões desènvolvidas nas seções anteriores consideram a presença do filtro de recepção da portadora interferida. Uma análise destas expressões permite concluir que nos casos de interesse o efeito deste filtro pode ser desprezado já que o mesmo não altera de forma significativa a porção do espectro do ruído de fase contida na faixa de freqüências do sinal multiplex da portadora FDM/FM.

Para os produtos de intermodulação correspondentes ao caso I isto pode ser facilmente verificado notando-se que:

i) em (46), a convolução $\bar{S}_{0}(f) *|\tilde{H}(f)|^{2}$ é essencialmente constante para freqüências inferiores à freqüência máxima $f_{m}$ do sinal multiplex da portadora FDM/FM, já que tanto $S_{O}(f)$ quanto $\tilde{H}(f)$ possuem faixa larga quando comparada à $f_{m}$. Deste modo, (46) pode ser aproximada por

$$
S_{\tilde{g}}^{+}(f)=A_{D}^{2} S_{B i k}^{+}(-f) K_{1} \quad, \quad|f| \leqslant f_{m}
$$

onde, admitindo-se que o filtro de recepção não distorce a portadora desejada,

$$
K_{1}=\int_{-\infty}^{\infty} \bar{S}_{0}(f)|\tilde{H}(f)|^{2} d f=1
$$

ii) um argumento similar pode ser utilizado com relação a $W(f)$, dado por (72), para aproximar (71) por

$$
S_{\tilde{g}_{1} \bar{g}_{2}}(f)=A_{D}^{2} e^{-j 2 \alpha_{D}} S_{B i k}^{\prime}(-f) \cdot K_{1} \quad,|f| \leqslant f_{m}
$$

Para os produtos de intermodulação correspondentes ao caso II, as seguintes observações podem ser feitas:

i) verifica-se, de (78), que como $S_{i}(f) \cdot$ e $S_{k}(f)$ correspondem aos espectros de portadoras de faixa estreita, $S_{B i k}(f)$ possui uma banda muito estreita quando comparada àquela de $Q^{\prime}(f)$ dado em (50). Deste modo para efeitos da integral em (49) o valor de $Q^{\prime}(f)$ pode ser considerado constante ao longo da faixa da freqüências de $S_{B i k}(f)$, e portanto (49) pode ser aproximada por 


$$
S_{\tilde{g}}^{-}(f)=A_{D}^{2} Q^{\prime}\left(f-\Delta f_{i k}^{-}\right) \int_{-\infty}^{\infty} S_{0}^{\prime}(f+\alpha) S_{B i k}^{-}(\alpha) d \alpha
$$

Considerando-se, por simplicidade, $\tilde{H}(f)$ um filtro retangular de lagura $B$, observa-se que quando $\left|\Delta f_{i k}^{-}\right|<B-f_{m} \circ$ valor de $O^{\prime}\left(f-\Delta f_{i k}^{-}\right)$é igual a $|\tilde{H}(0)|^{2}$ para freqüências $f$ tais que $|f| \leqslant f_{m}$ Deste modo, considerando-se $|\tilde{H}(0)|=1$, obtém-se a aproximação

$$
S_{\tilde{g}}^{-}(f)=A_{D}^{2}\left[S_{B i k}^{-}(-f) * S_{0}^{\prime}(f)\right]
$$

que é válida para $|f| \leqslant f_{m}$ e $\left|\Delta f_{i k}^{-}\right|=\left|f_{i}+f_{k}-2 f_{0}\right|<B-f_{m}$.

ii) os produtos de intermodulação centrados em freqüências $\Delta f_{i k}^{-}$superiores em módulo a $B-f_{m}$, para as quais a aproximação em (83) poderia não ser adequada, possuem uma potência desprezível na faixa de recepção do filtro. Nestes casos, tanto a forma exata quanto a aproximação em (83) são desprezíveis. Desta forma o uso de (83) para $\left|\Delta f_{i k}^{-}\right|>B-f_{m}$ não introduz erros significativos no resultado final em que todos os produtos são considerados.

\subsection{Determinação da NPR ("Noise-Power Ratio")}

A determinação da densidade espectral de potência em (63), relativa a pares de produtos de intermodulação correspondentes ao caso l, é feita a partir de (64), (81) e (82). Considerando-se que $S_{\tilde{g} 2}(f)$ em (64) é obtida invertendo-se os índices i e k em (81), e observando-se a partir de (78) que

$$
S_{B i k}^{+}(f)=S_{B i k}^{+}(-f)
$$

ob tém-se

$$
S_{\tilde{g}_{2}}(-f)=S_{\tilde{g}_{1}}(f)
$$

o que permite reescrever (64) como

$$
F(f)=\frac{1}{2 A_{D}^{4}} \operatorname{Re}\left\{S_{\tilde{g}_{1}}(f)-S_{\tilde{g}_{1} \tilde{g}_{2}}(f)\right\}=\frac{1}{2 A_{D}^{2}} \operatorname{Re}\left\{S_{B i k}^{+}(-f)-e^{-j 2 \alpha_{D}} S_{B i k}^{\prime}(-f)\right\}
$$

ou ainda, utilizando (78), (79) e (74),

$$
F(f)=\frac{1}{2 A_{D}^{2}} \hat{P}_{0 i k}\left[\bar{S}_{i} * \bar{S}_{k}\right]\left(f-\left(f_{i}-f_{k}\right)\right) ;|f| \leqslant f_{m}
$$


onde

$$
\hat{P}_{0 i k}=\operatorname{Re}\left\{P_{0 i k}-e^{-j 2 \alpha} P_{0 i k}^{\prime}\right\}
$$

A expressão (88) pode ser reescrita considerando-se (77), (80) e a identidade (12), na forma

$$
\hat{P}_{0 i k}=2 E\left\{\left[\operatorname{Im}\left(e^{-j \alpha_{D}} M(0, i, k, t)\right)\right]^{2}\right\}
$$

Utilizando-se a definição de $M($.$) em (30), obtém-se$

$$
\begin{aligned}
& \hat{P}_{0 i k}=2 p_{i} p_{k} \sum_{s=1}^{L} \sum_{n=1}^{L} \hat{b}_{s} \hat{b}_{n} J_{1}\left(\alpha s A_{0}\right) J_{1}\left(\alpha n A_{0}\right) J_{1}\left(\alpha s A_{i}\right) . \\
& . J_{1}\left(\alpha n A_{i}\right) . J_{1}\left(\alpha s A_{k}\right) J_{1}\left(\alpha n A_{k}\right) . \prod_{\substack{m \neq i, k \\
m \neq i}}^{M}\left[p_{m} J_{0}\left(\alpha_{s} A_{m}\right) J_{0}\left(\alpha n A_{m}\right)+\left(1-p_{m}\right)\right]
\end{aligned}
$$

onde

$$
b_{s}=\operatorname{Im}\left[e^{-j \alpha_{D}} b_{s}\right] \quad, \quad s=1, \ldots, L
$$

Para deteminar a densidade espectral de potência do ruído total de fase, em banda básica, é necessário que os espectros de cada parcela sejam ponderados por uma contagem seletiva de produtos de intermodulação, em tudo semelhante ao que é feito para a determinação do espectro de intermodulação em RF. Esta contagem seletiva pode ser obtida, por exemplo, através do algoritmo descrito em [6].

No caso de produtos correspondentes ao caso $I$, seja $n_{s, \ell}^{\prime}$, o número de produtos de intermodulação dependentes, de espécie $s[\mathbf{6}$ ], centrados na freqüência $\ell . \Delta f$, onde $\Delta f$ é a unidade de discretização da faixa de freqüências do transponder. $O$ índice s está restrito às espécies que envolvem a portadora FDM/FM.Seja $\varnothing 0$ conjunto destas espécies.

A densidade espectral de potência do ruído total de fase correspondente a produtos caracterizados pelo caso I pode ser escrita, utilizando-se (87) e (63), como

$$
S_{\eta}^{\prime}(f)=\sum_{\ell=0}^{L^{*}} \underset{s \bar{\epsilon}}{\sum} \prod^{n_{s, \ell^{*}+\ell}^{\prime}} \frac{\hat{P}_{s}}{2 A_{D}^{2}}\left[\bar{S}_{s}^{i}(f-\ell \Delta f)+\bar{S}_{s}^{\prime}(f+\ell \Delta f)\right], \quad|f| \leqslant f_{m}
$$


onde $\ell^{*}$ identifica a posição da portadora FDM/FM no transponder; $L^{*}$ é o menor inteiro maior que $B /(2 \Delta f)$, sendo $B$ a banda da portadora FDM/FM. A quantidade $\hat{P}_{S}$ é dada por $(\mathbf{9 0})$, com os índices i e $\mathrm{k}$ caracterizando os tipos das duas portadoras que, além da desejada, participam do produto de intermodulação de espécie s. O espectro normalizado $\bar{S}_{S}(f)$ é por sua vez dado pela convolução dos espectros normalizados dessas duas portadoras.

Uma vez determinada $S_{\eta}^{\prime}(f)$, a NPR devida a produtos correspondentes ao caso I, no i-ésimo canal da banda básica da portadora FDM/FM é dada por

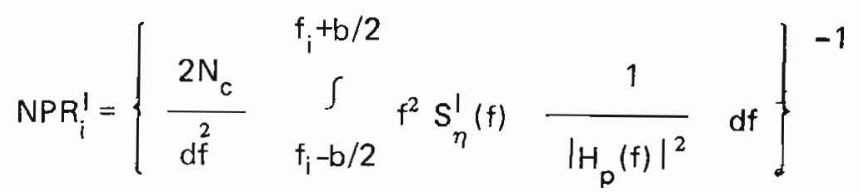

onde $d^{2}{ }_{f}$ é o desvio médio quadrático da portadora $F D M / F M ; N_{c}$ é o número de canais de voz do sinal multiplex; b é a banda de um canal de voz e $f_{i}$ a freqüência central do i-ésimo canal. $O$ filtro $H_{p}(f)$ corresponde à característica de pré-ênfase normalizada utilizada.

A determinação da densidade espectral de potência relativa a um produto de intermodulação correspondente ao caso II, pode ser obtida combinando-se (83) e (78) resultando

$$
S_{\tilde{g}}^{-}(f)=A_{D}^{2} P_{0 i k}\left[S_{0}^{\prime} * \bar{S}_{i} * \bar{S}_{k}\right]\left(f+f_{i}+f_{k}-2 f_{0}\right) \quad, \quad|f| \leqslant f_{m}
$$

De forma análoga ao que foi descrito para o caso I, a densidade espectral de potência do ruído total de fase correspondente a produtos caracterizados pelo caso II pode ser escrita, utilizando-se (94) e (14a), como

$S_{\eta}^{\prime \prime}(f)=\sum_{\ell=-L^{* *}}^{L^{* *}} \sum_{s \in}^{\Sigma} \oslash \eta_{s, \ell^{*}+\ell}^{\prime \prime} \frac{P_{s}}{4 A_{D}^{2}}\left[\bar{S}_{s}^{\prime \prime}(f-\ell \triangle f)+\bar{S}_{s}^{\prime \prime}(f+\ell \triangle f)\right],|f| \leqslant f_{m}$

onde $\ell^{*}$ indica a posição da portadora FDM/FM no transponder; $L^{* *}$ é o menor inteiro maior que $(B+f) / \Delta f$ sendo $B$ a banda da portadora FDM/FM e $\eta_{s, \ell}^{\| \prime}$ é número de produtos de intermodulação dependentes, de espécie $s$, centrados na freqüência $\ell . \Delta f$, sendo $\Delta f$ a unidade de discretização da faixa de freqüências do transponder. A quantidade $P_{S}$ em (95), obtida a partir de (77) e (32), é dada por

$J_{\text {oik }}=p_{i} p_{k} \sum_{s=1}^{L} \sum_{n=1}^{L} b_{s}^{*} b_{n} J_{1}\left(\alpha s A_{0}\right) J_{1}\left(\alpha n A_{0}\right) J_{1}\left(\alpha_{s} A_{i}\right) J_{1}\left(\alpha_{n} A_{i}\right) . J_{1}\left(\alpha_{s} A_{k}\right) J_{1}\left(\alpha n A_{k}\right)$. 


$$
\prod_{\substack{m=1 \\ m \neq i, k}}^{M}\left[p_{m} J_{0}\left(\alpha_{s} A_{m}\right) J_{0}\left(\alpha_{n} A_{m}\right)+\left(1-p_{m}\right)\right]
$$

com os índices i e $\mathrm{k}$ caracterizando os tipos das duas portadoras que, além da desejada, participam dos produtos de intermodulação de espécie's. O espectro normalizado $\bar{S}_{s}^{\prime \prime}(f)$ é dado pela convolução dos espectros destas duas portadoras e do espectro expandido, $S_{0}^{\prime}(f)={ }_{2} \bar{S}_{0}\left(\frac{f}{2}\right)$, da portadora desejada.

Uma vez determinado $S_{\eta}^{\prime \prime}(f)$, a NPR devida a produtos correspondentes ao caso II, no i-ésimo canal da banda básica da portadora FDM/FM, é obtida substituindo-se $S_{\eta}^{\prime}(f)$ por $S_{\eta}^{\prime \prime}(f)$ em (93).

\subsection{Extensão dos Resultados ao Caso Não Gaussiano}

As expressões para densidade espectral de potência do ruído de fase utilizadas na seção anterior na determinação da NPR (caso I e caso II) foram obtidas a partir de expressões aproximadas para as quantidades fundamentais $\mathrm{S}_{\tilde{g}}^{+}(f), S_{\tilde{g}_{\tilde{g}} \tilde{g}_{2}}(f)$ e $S_{\tilde{g}}^{-}(f)$ dadas na subesção 4.4. Estas expressões foram por sua véz obtidas a partir das expressões gerais de $R_{\tilde{g}}^{ \pm}(\tau)$ e $\mathbb{R}_{\tilde{g}_{1}} \tilde{g}_{2}(\tau)$. dadas por (37) e (65), da seguinte maneira. Em primeiro lugar considerou-se Gaussiano o processo que caracteriza o sinal multiplex da portadora FDM/FM, - que permitiu expressar $R_{\tilde{g}}^{ \pm}(\tau)$ e $R \tilde{g}_{1} \tilde{g}_{2}(\tau)$ por (39) e (67). Em seguida, a suposição de um espectro de forma Gaussiana para a portadora FDM/FM permitiu a obtenção, em forma fechada, dos espectros $S_{\tilde{g}}^{+}(f), S_{\tilde{g}}^{-(f)}$ e $S_{\tilde{g}_{1}} \tilde{g}_{2}(f)$ associados às funções autocorrelação e correlação cruzada acima e dadós respectivamente por (46), (49) e (71). Uma análise destas últimas expressões mostrou que, para portadoras FM de faixa larga, a influência do filtro de recepção da portadora desejada não é significativa, conduzindo finalmente às aproximações (81), (82) e (83) da subseção 4.4.

A conjectura de que o filtro de recepção da portadora desejada não altera significativamente o espectro do ruído na faixa de interesse parece ser razoável mesmo para portadoras de faixa larga de espectro nâo necessariamente Gaussiano. Isto sendo verdade, os resultados da subseção 4.4 são também aplicáveis ao caso não-Gaussiano. Esta afirmação pode ser facilmente verificada, eliminando-se a priori o filtro de recepção, ou seja, fazendo-se nas expressões gerais (37) e (65)

$$
h(\alpha)=\delta(\alpha) \text { e } h(\beta)=\delta(\beta) .
$$

Com esta substituição, resulta imediatamente de (37)

$$
R_{\tilde{g}}^{+}(\tau)=A_{D}^{2} R_{B i k}^{+}(-\tau)
$$


e

$$
R_{\tilde{g}}^{-}(\tau)=A_{D}^{2} R_{B i k}^{-}(-\tau) E\left\{\exp \left[2 j\left(\theta_{0}(t+\tau)-\theta_{0}(t)\right)\right]\right\}
$$

e de $(65)$

$$
R_{\tilde{g}_{1} \tilde{g}_{2}}(\tau)=A_{D}^{2} e^{-j 2 \alpha_{D}} R_{B i k}^{\prime}(-\tau)
$$

As transformadas de Fourier de (97) e (99) são exatamente as aproximações dadas em (81) e (82). A transformada de (98) por sua vez é a expressão em $(\mathbf{8 3})$, onde $S_{0}^{\prime}(f)$ corresponde à densidade espectral de potência normalizada da portadora FDM/FM com o dobro do desvio r.m.s. de freqüência (no caso de espectro Gaussiano, $S_{0}^{\prime}(f)=\frac{1}{2} \bar{S}_{0}\left(\frac{f}{2}\right)$ ). Note-se que para obter estes resultados não foram necessárias hipóteses Gaussianas nem para o sinal multiplex nem para o espectro FDM/FM, sendo necessária apenas a hipótese de que o filtro de recepção da portadora desejada pode ser desprezado.

\section{Exemplo}

O presente exemplo considera a situação particular apresentada na Fig. 1(a) onde uma portadora FDM/FM compartilha um mesmo transponder com 500 portadoras SCPC/FM ativadas por voz, e de mesma potência. As características das portadoras envolvidas são as seguintes.

(i) Portadora FDM/FM

- número de canais: 312

- largura de faixa alocada: $15 \mathrm{MHz}$

- largura de faixa ocupada: 13,5 $\mathrm{MHz}$

- Freqüência máxima da banda básica: 1,3 $\mathrm{MHz}$

- desvio r.m.s. multicanal: 1716 kHz

- e.i.r.p. ("equivalent isotropically radiated power"): 64, 5 dBW

- espectro aproximado por curva Gaussiana.

(ii) Portadora SCPC/FM com ativação por voz

- coeficiente de ativação por voz: 0,4

- espectro apresentado na Fig. 2, gerado de acordo com[7]e com as seguintes características:

- nível de sinal de voz aleatório

- média "long term" do nível do sinal de voz: $-20 \mathrm{dBm0}$

- desvio padrão do nível do sinal de voz: 5,8 dB

- desvio r.m.s. do tom de teste: $5,2 \mathrm{kHz}$

- espaçamento entre as portadoras: $30 \mathrm{kHz}$

- e.i.r.p. (quando ativadas): $37 \mathrm{dBW}$ 


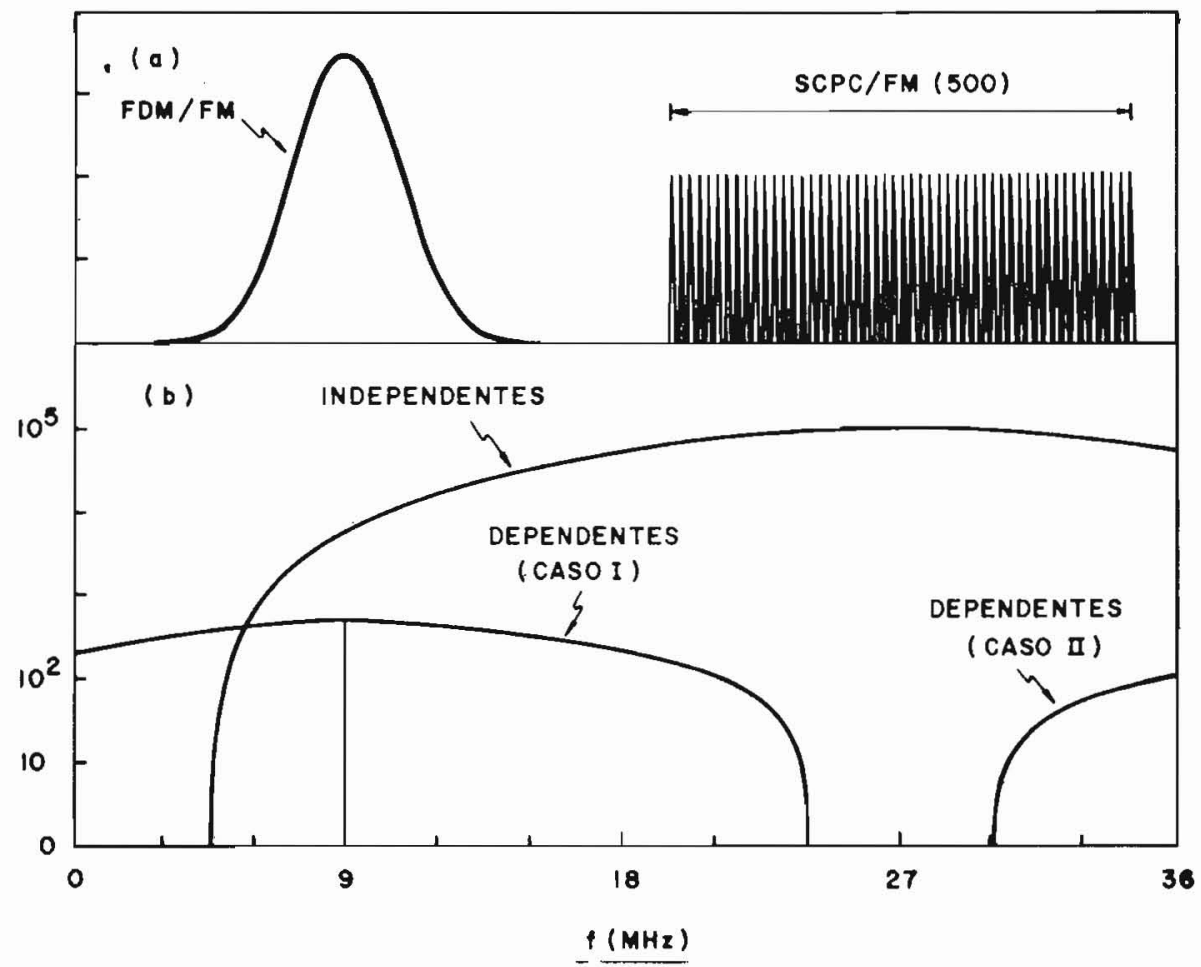

Figura 1. (a) Configuração do transponder; (b) contagem dos produtos de intermodulação de terceira ordem do tipo $f_{a}+f_{b}-f_{c}$.

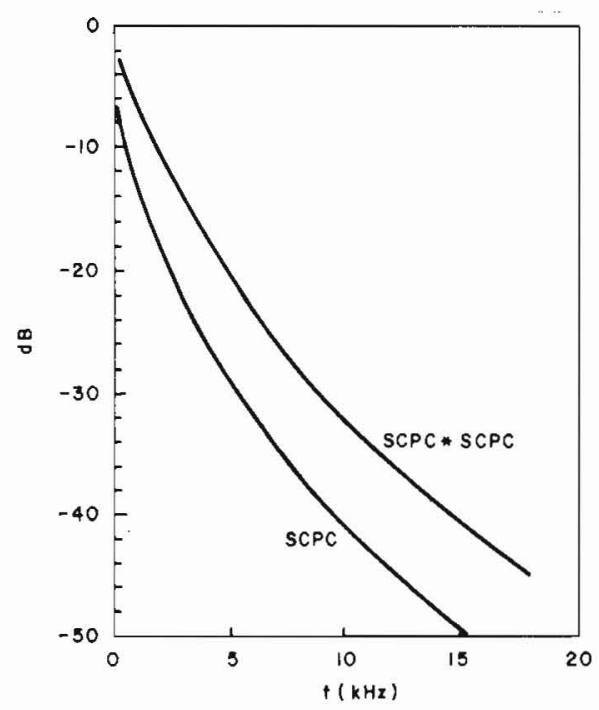

Figura 2. Espectro de uma portadora SCPC/FM e aquele correspondente à convolução de dois espectros de portadoras SCPC/FM. 
A característica não-linear da TWT foi dada pela expressão usual em funções de Bessel[3], com coeficiantes característicos das TWT's do INTELSAT IV [5]. Para portadoras com as características apresentadas em (i) e (ii) considerou-se um "back-off" típico de 13,7 dB.

O Algarismo Rápido de Contagem ARC [6] foi utilizado para dete minar a contagem seletiva dos produtos de intermodulação de $3{ }^{a}$ ordem, do tipo $f_{a}+f_{b}-f_{c}$. Para a aplicação do ARC, a faixa de freqüências do transponder foi discretiza$\mathrm{da}$ em intervalos iguais ao espaçamento das portadoras SCPC/FM (30 kHz). Foram contados os produtos de intermodulação independentes da portadora desejada e os dependentes (caso I e caso II). O resultado desta contagem seletiva é ilustrado na Fig.1(b). Observa-se que conforme previsto na subseção 4.1, para a configuração em questão os produtos correspondentes ao caso II são totalmente rejeitados pelo filtro de recepção da portadora FDM/FM e por este motivo não foram considerados no cálculo do ruído de intermodulação em banda básica.

Para a determinaçāo do espectro do ruído de freqüência a partir do espectro do ruído de fase, deve ser levado em conta, além do diferenciador, o filtro de de-ênfase utilizado. No presente exemplo, considerou-se a característica de pré-ênfase dada pela Recomendação 464-1 do CCIR[8]. A Fig. 3 apresenta a característica combinada dos efeitos do diferenciador e do circuito de de-ênfase.

A forma da densidade espectral do ruído de freqüência devido aos produtos de intermodulação (caso I), obtido a partir de (92), incluindo-se a característica de freqüência da Fig. 3 é apresentada na Fig. 4. Observa-se que para a situação particular de que trata este exemplo, apenas uma espécie de produto dependente deve ser considerada: aquela que envolve duas portadoras SCPC/FM e a portadora FDM/FM. Além disso, o espectro $S_{s}(f)$ em (92) é dado pela convolução dos espectros de duas portadoras SCPC/FM. Este espectro encontra-se também ilustrado na Fig. 2, e é o principal responsável pelas variações acentuadas na amplitude do espectro do ruído de freqüência que aparece na Fig. 4.

Quando a dependência entre os produtos da intermodulação e o sinal desejado é ignorada, o espectro do ruído de fase em banda básica, gerado pelos produtos de intermodulação dependentes, pode ser obtido a partir de (92), $\mathrm{comS}_{s}^{\prime}$ (f)representando o espectro obtido através da convolução de quatro especiros (2 correspondentes à portadora SCPC/FM e 2 correspondentes à portadora $F D M / F M), \hat{P}_{S}$ substituído por $P_{S}$ obtido de (96), e $L^{*}$ estendido para 0 menor inteiro maior que $\left(B+f_{m}\right) / \Delta f$. $O$ espectro do ruído de fase resultante é essencialmente plano na faixa de freqüências de interesse. $A$ forma espectral do ruído de freqüência correspondente, incluindo o efeito de de-ênfase, é apresentado na Fig. 5. 


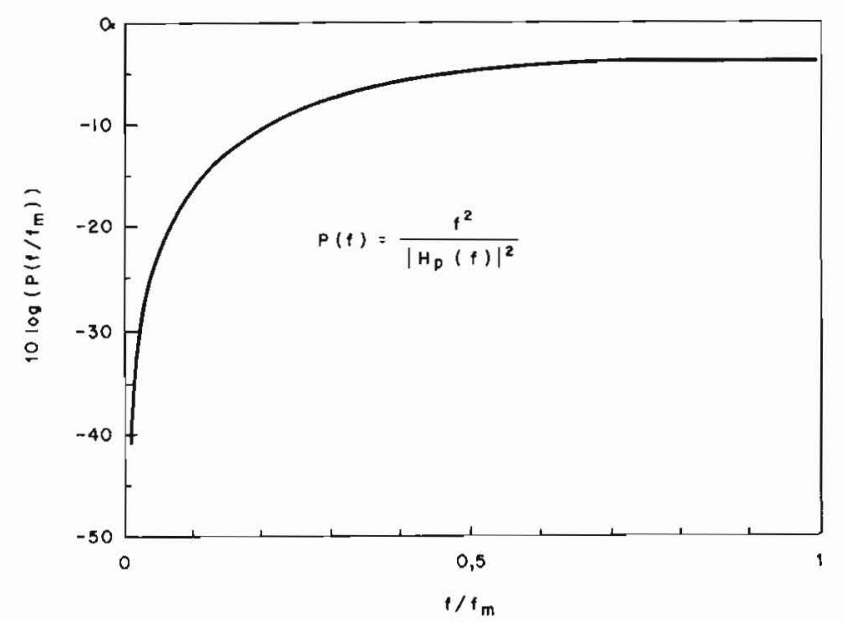

Figura 3. Característica combinada dos efeitos do diferenciador e do circuito de de-ênfase.

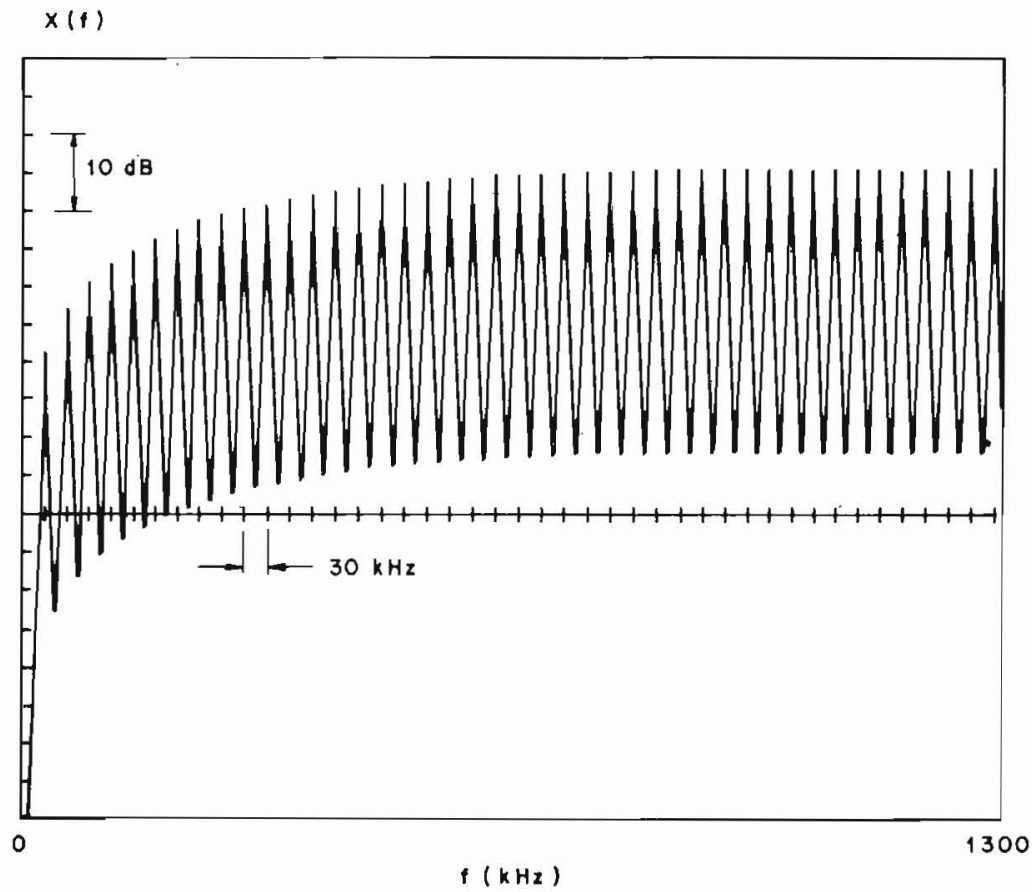

Figura 4. Forma da densidade espectral do ruído de freqüência na banda básica da portadora FDM/FM, devido a produtos de intemodulação dependentes (caso I), incluindo o efeito da de-ênfase. 
Convém ressaltar que o objetivo das figuras 4 e 5 é apenas o de ilustrar as variações dos espectros nelas apresentados e por estes motivo as amplitudes indicadas não representam valores absolutos.

No caso dos produtos de intermodulação independentes da portadora FDM/ FM, ou seja, aqueles gerados por três portadoras SCPC/FM, o espectro do ruído de fase foi obtido a partir de uma equação análoga a (95), utilizando-se obviamente a contagem relativa aos produtos independentes e as potências correspondentes. Além disso, o espectro $\bar{S}_{s}^{\prime \prime}$ (f) foi substituído

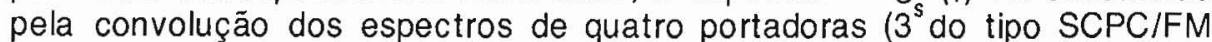
e 1 do tipo FDM/FM). Assim como no caso anterior, este espectro de fase resultou essencialmente plano, e a correspondente forma espectral para o ruído de freqüência é a mesma da Fig. 5.

Foi determinada finalmente, para cada canal do sinal multiplex (banda $b=4 \mathrm{kHz}$ ) da portadora FDM/FM, a razão sinal-ruído de intermodulação devida a todos os produtos de intermodulação de terceira ordem dependentes e indepen-

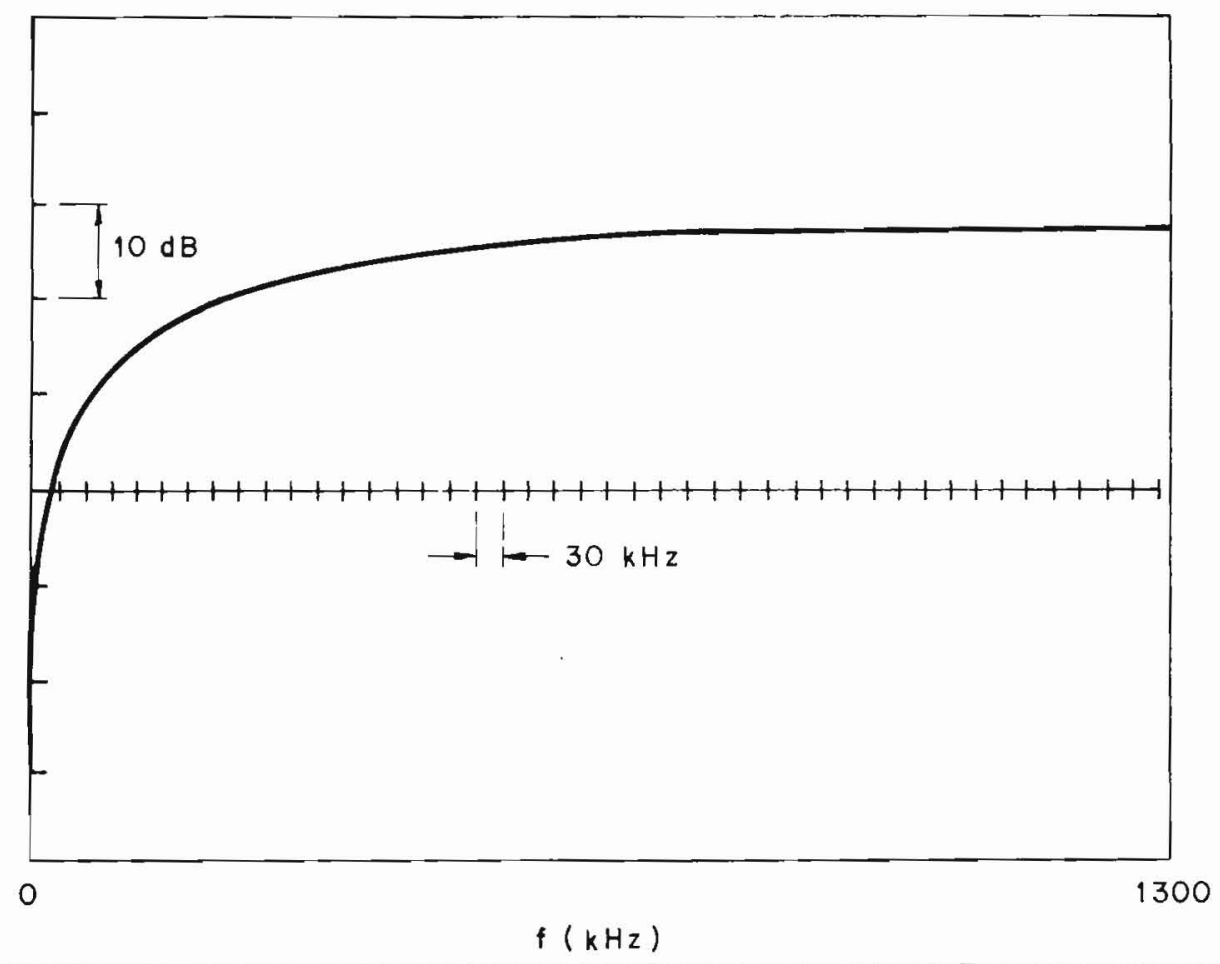

Figura 5. Forma da densidade espectral do rú́do de freqüência na banda básica da portadora FDM/FM, devido a produtos de intermodulação dependentes, quando esta dependência é ignorada.

Revista da Socledade Braslleira de Telecomunicaçð̌es 
dentes do tipo $f_{a}+f_{b}-f_{c}$. Estes valores foram determinados a partir de dois procedimentos distintos. No primeiro, os produtos de intermodulação dependentes foram tratados adequadamente, e no segundo a dependência destes produtos foi ignorada. A Fig. 6 apresenta os resultados obtidos.

Estes resultados mostram que, ao se considerar a dependência dos produtos de intermodulação com a portadora desejada, chega-se, no caso dos canais mais sacrificados, a valores de razão sinal-ruído de intermodulação até 8 $\mathrm{dB}$ menores que aqueles que seriam previstos por uma análise que não considera esta dependência.

É importante ressaltar que a diferença entre os valores de NPR previstos por intermédio de cada uma das análises (dependência considerada e dependência não considerada) é devida não só a diferentes formas para a densidade espectral do ruído de frequeência, conforme ilustrado pelas figuras 4 e 5 , mas também ao fato de que as potências associadas a estes espectros podem diferir significativamente. Note que para uma análise que não considere a dependência entre os sinais interferido e interferente, a potência associada

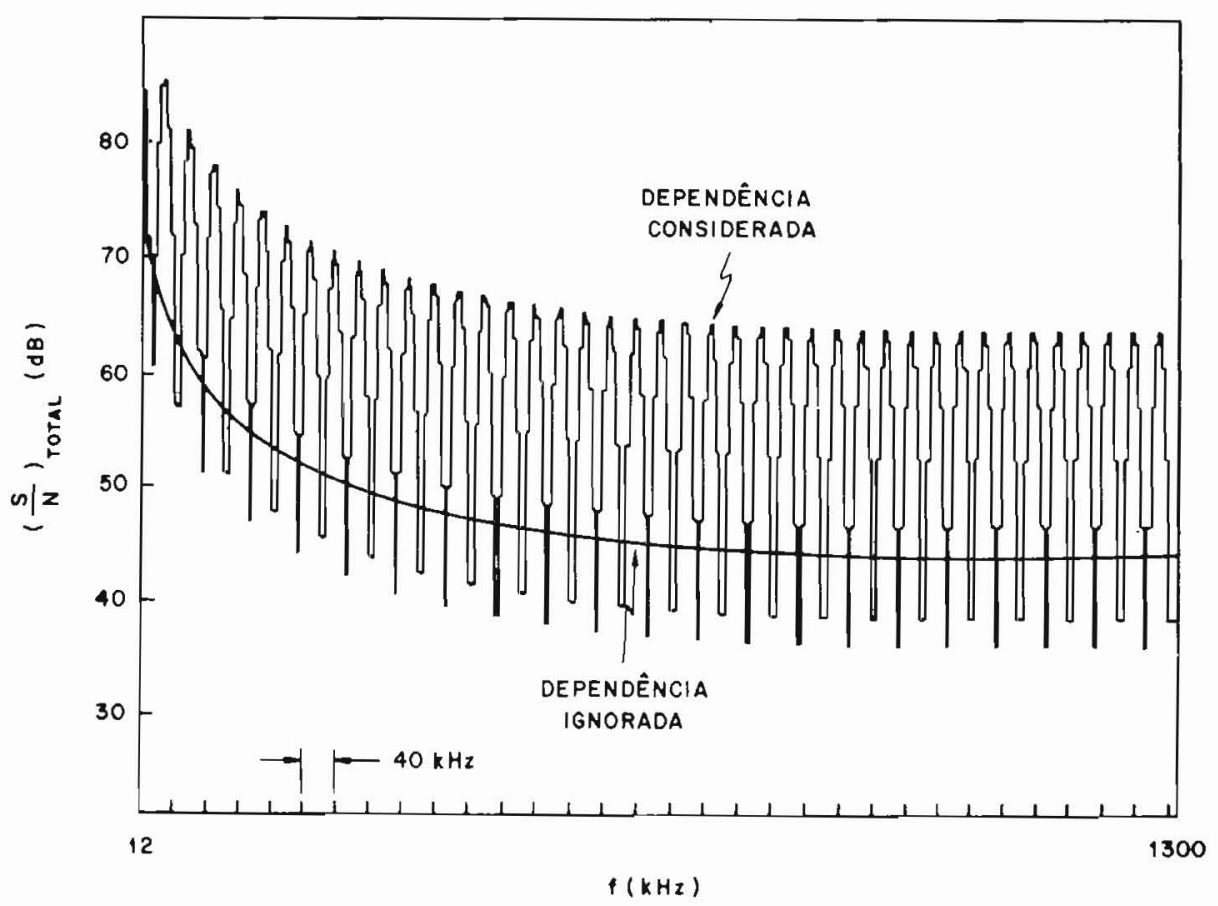

Figura 6. Razão sinal-ruído total de intemodulação em cada canal de 4 $\mathrm{kHz}$ da banda básica da portadora FDM/FM. 
ao espectro do ruído em banda básica gerado pelos produtos de intermodulação incluídos no caso I varia apenas com o módulo da amplitude complexa do produto considerado. Entretanto, é evidente de (89) que esta potência varia não só com o módulo mas também com a fase da amplitude complexa do produto de intermodulação (relativa à fase da amplitude complexa da portadora desejada). Como esta defasagem relativa é também função do ponto de operação do dispositivo não-linear, as diferenças entre os valores de NPR previstos pelas duas análises podem ser mais, ou menos, pronunciadas dependendo do "black-off" utilizado.

\section{Conclusões}

O presente trabalho apresentou uma análise detalhada dos efeitos da intermodulação gerada na banda básica de uma portadora FDM/FM, que compartilha uma não-linearidade com várias outras portadoras de faixa estreita. As dificuldades analíticas inerentes ao problema consideradas no presentes trabalho envolvem a ativação por voz das portadoras SCPC, a dependência estatística entre os sinais interferente e interferido, a filtragem da intermodulação pelo filtro de recepção da portadora desejada e a correlação cruzada entre as parcelas de ruído geradas em banda básica. Todos estes aspectos foram cuidadosamente levados em consideração e expressões em forma fechada foram obtidas para os espectros do ruído de fase na banda básica da portadora FDM/FM. Um análise dessas expressões gerais permitiu que se visualizassem condições sob as quais a influência do filtro de recepção da portadora FDM/ FM pode ser desprezada.

No caso de intermodulação dependente, cumpre ressaltar a existência de dois tipos de espectro para o ruído de fase, cujos efeitos devem ser analisados separadamente. Um espectro com variações suaves, produzido pelos produtos de intemodulação aqui enquadrados no caso II, e outro que pode ter variações bastante acentuadas já que é formado apenas a partir dos espectros das portadoras de faixa estreita e que é devido aos produtos de intermodulação correspondentes ao caso I. Ressalte-se ainda que a potência associada a este segundo tipo de espectro depende não só do módulo mas também da fase da amplitude complexa dos produtos de intermodulação, relativa àquela da portadora desejada.

Em situações como a considerada no exemplo que aparece na Seção 5, somente o segundo tipo de espectro existe na faixa da portadora FDM/FM. Como neste caso particular a contribuição dos produtos de intermodulação dependentes é dominante, obtém-se variações acentuadas de NPR ao longo dos canais da banda básica. Tais variações não seriam detectadas, caso a dependência de produtos de intermodulação com a portadora desejada fosse ignorada, levando a erros bastante significativos nas estimativas de NPR. 
Embora as expressões finais apresentadas na subseção 4.5 tenham sido obtidas com a ajuda de aproximações Gaussianas para o sinal modulador e para o espectro da portadora FDM/FM (bastante realistas e usuais em vários casos de interesse), foi discutida na subseção 4.6 a aplicação destas mesmas expressões ao caso mais geral que considera portadoras FDM/FM de faixa larga com espectro não necessariamente Gaussiano.

\section{Referências}

[1] N. K. M. Chitre e J. C. Fuenzalida, "Baseband Distortion Caused by Intermodulation in Multicarrier FM Systems", COMSAT Technical Review, vol. 2, n. ${ }^{\circ}$, Spring 1972, pp. 147-172.

[2] B. A. Pontano, J. C. Fuenzalida e N. K. M. Chitre, "Interference into Angle Modulated Systems Carrying Multichannel Telephony Signals", IEEE Transactions on Communications, vol. COM-21, n. ${ }^{\circ}$, Junho 1973, pp. 714-727.

[3] J. C. Fuenzalida, O. Shimbo e W. L. Cook, "Time-Domain Analysis of Intermodulation Effects Caused by Nonlinear Amplifiers", COMSAT Technical Review, vol. 3, n. 1, Spring 1973, pp. 89-143.

[4] R. Sampaio Neto, "Efeitos de Intermodulação em Sistemas SCPC/FM com Ativação por Voz", Publicação CETUC-D-ST-101/78, Julho 1978.

[5] R. Sampaio Neto e J. M. Fortes, "Intermodulação sobre Portadora FDM/ FM que Compartilha o Transponder com Portadoras de Faixas Estreitas", Publicação CETUC-D-ST-02/87, Janeiro 1987.

[6] J. M. Fortes e R. Sampaio Neto, "A Fast Algorithm for Sorting and Counting Third-Order Intermodulation Products", IEEE Transactions on Communications, vol. COM-34, n. ${ }^{\circ} 12$, Dezembro 1986, pp. 1266-1272; e "Um Algoritmo Rápido para a Contagem de Produtos de Intermodulação", Anais do 3.: Simpósio Brasileiro de Telecomunicações, S. J. dos Campos, SP, Setembro 1985, pp. 178-184.

[7] R. Sampaio Neto e J. P. Albuquerque, "Intermodulation Effects in the Transmission of Voice - Activated SCPC/FM Carriers Through a Nonlinear Repeater", IEEE Transactions on Communications, vol. COM-29, n. ${ }^{\circ} 10$, Outubro 1981, pp. 1537-1547.

[8] "Pre-Emphasis Characteristics for Frequency - Modulation Systems for Frequency-Division Multiplex Telephony in the Fixed-Satellite Service", Recomendação 464-1 do CCIR, 16. ${ }^{a}$ Assembléia Plenária, Dubrovnik, 1986. 


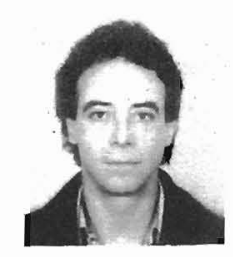

RAIMUNDO SAMPAIO NETO nasceu em Resende, Rio de Janeiro, em 16 de junho de 1952. Formou-se em Engenharia Elétrica - Telecomunicações na PUC/Rio, em 1975, e obteve o grau de Mestre em Ciências em Engenharia Elétrica na mesma Universidade em 1978. Concluiu o doutorado na University of Soüthern California (USC) em Los Angeles, em 1983, com o título de PhD em Engenharia Elétrica. De 1983 a 1984 prestou serviços de consultoria, em análise de sistemas de comunicações, junto a Axiomatic Inc, em Los Angeles, e desenvolveu atividades de pesquisa na área de sincronização de códigos em sistemas "spread-spectrum" como Post-doctoral Fellow do Communication Sciences Institute da USC. Em julho de 1984 retornou à PUC/Rio onde é atualmente Professor Associado, trabalhando em ensino e pesquisa no Centro de Estudos em Telecomunicações da Universidade Católica (CETUC). Suas principais áreas de interesse são comunicações via satélite, sincronização em sistemas digitais e sistemas "spread-spectrum".

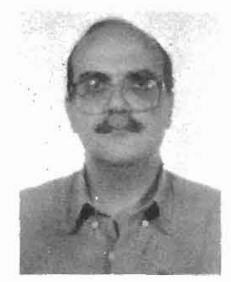

JOSÉ MAURO P. FORTES nasceu no Rio de Janeiro, em 25 de maio de 1950. Formou-se em Engenharia Elétrica-Telecomunicações na PUC/Rio em 1973 e obteve o grau de Mestre em Ciências em Engenharia Elétrica na mesma Universidade em 1976. Concluiu o doutorado na Stanford University em 1980 com o título de PhD em Engenharia Elétrica. Em junho de 1980 retornou à PUC/Rio onde é atualmente Professor Associado, trabalhando em ensino e peșquisa no Centro de Estudos em Telecomunicações (CETUC). E coordenador da Comissão Brasileira de Radiocomunicações para o Serviço Fixo por Satélite (CBR4) e vice-presidente da Comissão de Éstudos 4 do Comitê Consultivo Internacional de Radiocomunicaçōes (CCIR). Seus interesses atuais são nas áreas de comunicações por satélite, sincronização e processamento digital de imagens. 